\title{
In Situ TEM Observation of Domain Propagation and Relaxation Kinetics in Bismuth Ferrite Under Applied DC Bias
}

\author{
C.R. Winkler, ${ }^{*}$ L.W. Martin, ${ }^{* *}$ and M.L. Taheri* \\ * Department of Materials Science \& Engineering, Drexel University, Philadelphia, PA 19104 \\ ** Department of Materials Science and Engineering, University of Illinois at \\ Urbana-Champaign, Urbana, IL 61801
}

Select multiferroic materials exhibit ferroelectric and magnetic order, and the two order parameters are coupled through a quantum-mechanical exchange interaction [1,2]. One of the most widely studied magneto-electric multiferroics is the perovskite $\mathrm{BiFeO}_{3}(\mathrm{BFO})$. The magneto-electric coupling in $\mathrm{BFO}$ allows control of the ferroelectric and magnetic domain structures via applied electric fields. Furthermore, BFO films possess high Néel and Curie temperatures and large ferroelectric polarization. Because of these unique properties, BFO and other magneto-electric multiferroics constitute a promising class of materials for incorporation into devices such as high-density ferroelectric and magnetoresistive memories, spin valves, and magnetic field sensors [3,4]. However, the magneto-electric coupling in BFO is mediated by volatile ferroelastically switched domains that make it difficult to incorporate this material into devices. To facilitate device integration, an understanding of the microstructural factors that affect ferroelastic relaxation and ferroelectric domain switching must be developed.

We employ a custom-built biasing holder which permits the application of a DC bias through a set of epitaxial in-plane electrodes in the BFO thin film. By altering the magnitude and the polarity of the DC bias, we can control the magnitude and switch the direction of the in-plane electric field. We investigate the evolution of ferroelastically switched ferroelectric domains in BFO thin films during many switching cycles using in-situ TEM. We present evidence of domain nucleation, propagation, and switching even at applied electric fields below the coercive field (Figure 1). Quantitative kinetic data is extracted from these in situ videos (Figure 2). These observations indicate that the occurrence of ferroelastic relaxation in switched domains and the stability of these domains is influenced by the local microstructure of the BFO film. These biasing experiments provide a real time view of the complex dynamics of domain switching and complement scanning probe techniques $[5,6]$.

References

[1] R. Ramesh \& N.A. Spaldin, Nature Mater. 6 (1), (2007) 21.

[2] C. Ederer \& N.A. Spaldin, Curr. Opin. Solid State Mater. Sci. 9 (3), (2005) 128.

[3] Y.H. Chu et al., Nature Mater. 7 (8), (2008) 678.

[4] N.A. Spaldin \& M. Fiebig, Science 309 (5733), (2005) 391.

[5] S.H. Baek et al., Nature Mater. 9, (2010) 309.

[6] This research was made possible by use of the Centralized Research Facilities in the College of Engineering at Drexel University. This work was supported by the Department of Education and Drexel University through the GAANN-DREAM fellowship under contract P200A060117. 


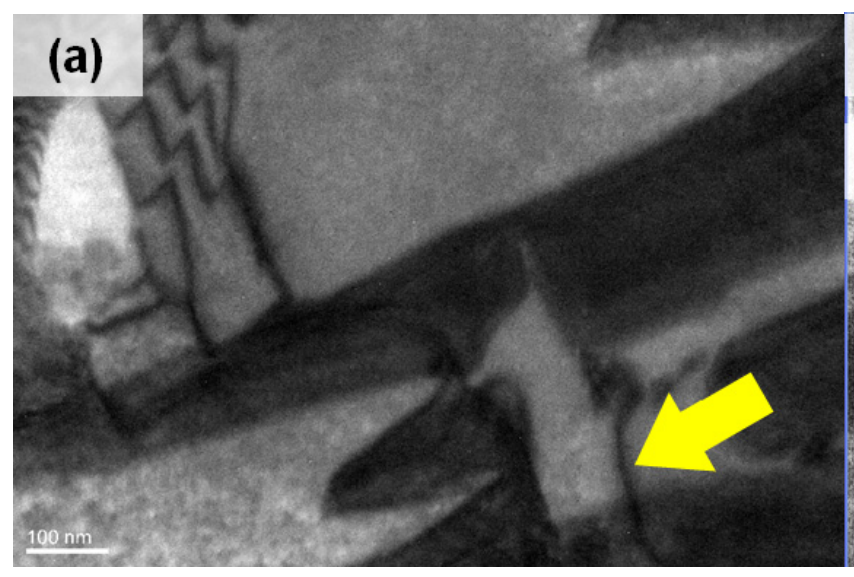

\section{Zero field}

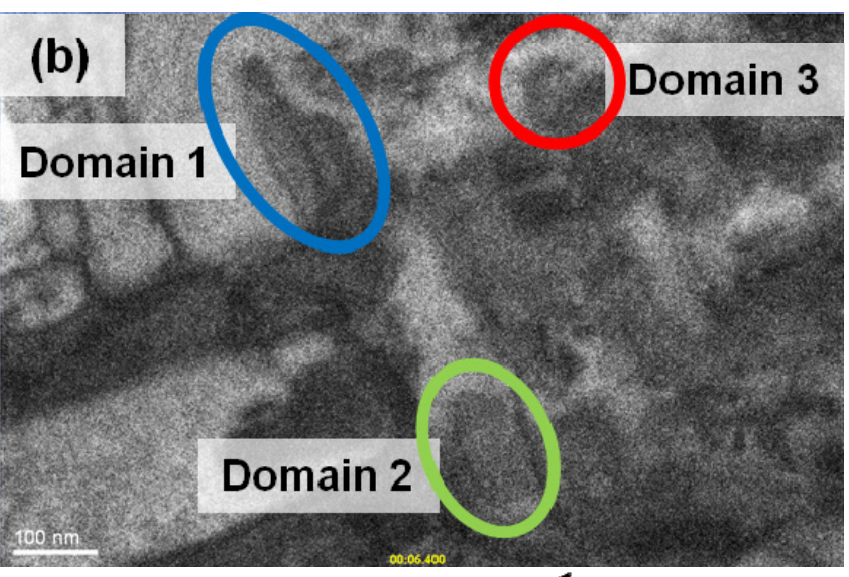

$E \sim 150 \mathrm{kV} / \mathrm{cm}$

Figure 1 - The TEM image in (a) depicts the configuration of domains, the finger-like structures with enhanced contrast, prior to application of $+45 \mathrm{~V}$ in (b). The yellow arrow in (a) indicates a visible dislocation. In (b), an image captured less than $100 \mathrm{~ms}$ after applying a $150 \mathrm{kV} / \mathrm{cm}$ electric field shows newly-formed domains growing orthogonal to existing domain walls before later fully relaxing. Domain area over time is calculated for three specific domains, each of which exhibits different behavior.

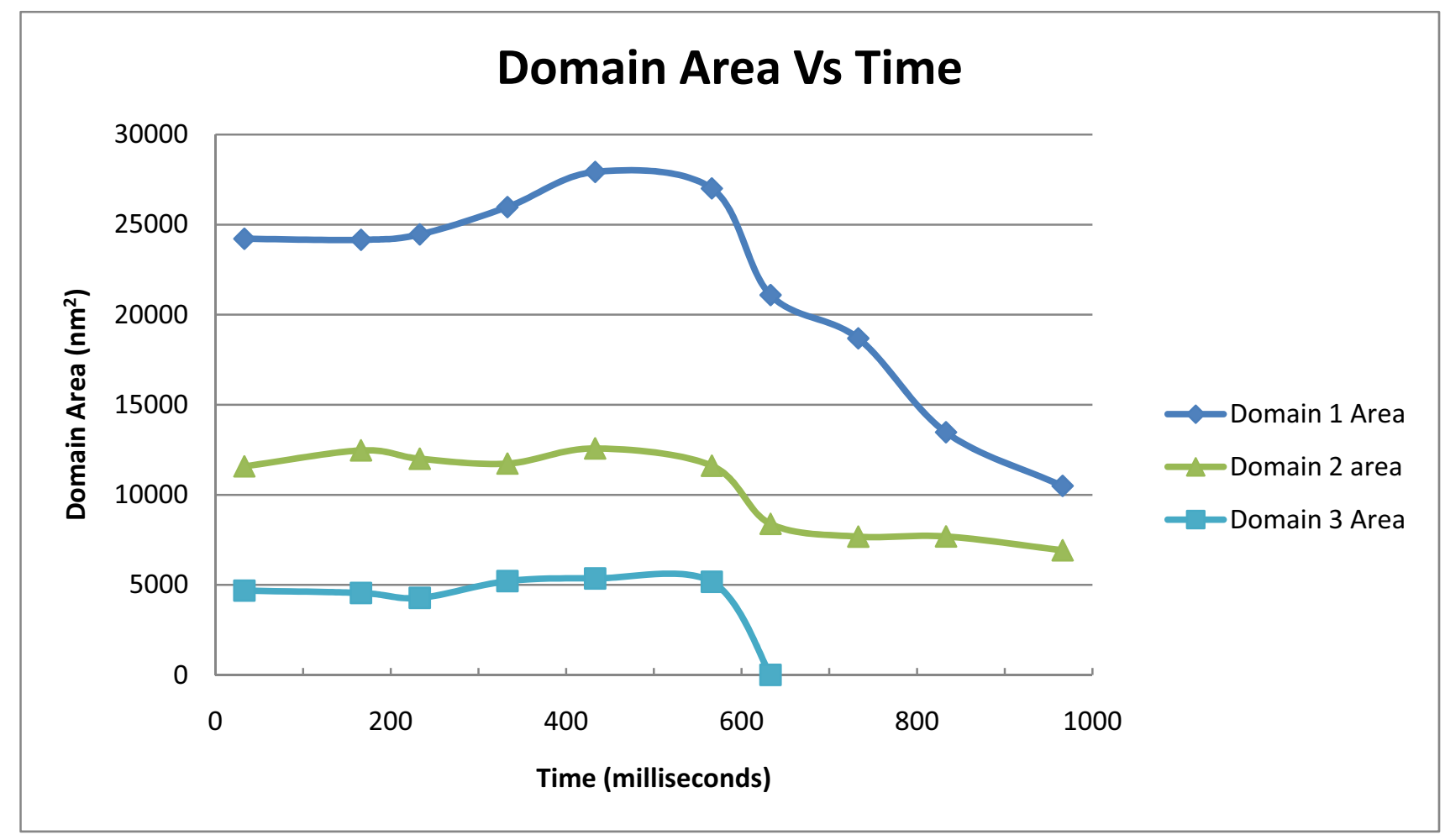

Figure 2 - Differing relaxation behavior is evident for the selected domains depicted in $\mathbf{1}(\mathrm{b})$. Domain 1 is observed to expand both parallel and perpendicular to its growth direction under the applied field before gradually fully relaxing, whereas Domain 3 rapidly relaxes after removal of the field. Domain $\mathbf{2}$, however, is stabilized against relaxation by the dislocation highlighted in Figure 1(a). 